\title{
Are current flow models for transcranial electrical stimulation fit for purpose?
}

Sven Bestmann, Nick Ward

Sobell Department for Motor Neuroscience and Movement Disorders, UCL Institute of Neurology, 33

Queen Square, London, WC1N3BG, UK.

Email: s.bestmann@ucl.ac.uk

Key words: Validation; variability; tDCS; dose-control; optimization

Transcranial electrical stimulation (tES) enjoys a burgeoning reputation in basic and clinical research, and home-use. Even though Rush and Driscoll highlighted more than 40 years ago that "the amount of current entering the brain is of great consequence"[9], to this day tES studies generally do not control how much current is actually delivered to the brain. Instead, tES applications control the output of a stimulator, and so for most applications we do not control that comparable doses of current are delivered to the brain of different individuals. Yet we do know that the idiosyncratic properties of the head strongly influence how much of a fixed output current (e.g. $1 \mathrm{~mA}$ ) will reach the brain [1], and so we are in the extraordinary position of knowing that the effective dose of tES is highly variable across individuals (Figure 1), yet doing very little about it.

The recent debate scrutinizing the efficacy, reliability and utility of tES $[4,5,10]$ thus misses a vital point that likely contributes to variable outcomes. For a given stimulator output, current in a cortical target region may vary by up to $100 \%$ across individuals[7]. For the field to mature, and the clinical promise of tES to be rigorously tested, not knowing how much current we deliver or where this current might travel, will remain an unacceptable barrier. This is especially the case in clinical applications such chronic stroke where results have been rather mixed[3], and where the distortions of current flow that the lesions impose may amplify variability in effectively delivered current. 
One solution to this problem is current flow modelling, which provides numerical estimates of current flow for a given electrode montage in an individual brain, based on MRI scans [1]. Models differ in their level of detail, but common to all is their reliance on approximations about the conductivity of different tissue types. These models have inspired the development of novel electrode montages[2]. Surprisingly, however, until recently we did not know whether these models are accurate in predicting current flow in an individual.

\section{A \\ Fixed stimulator output}

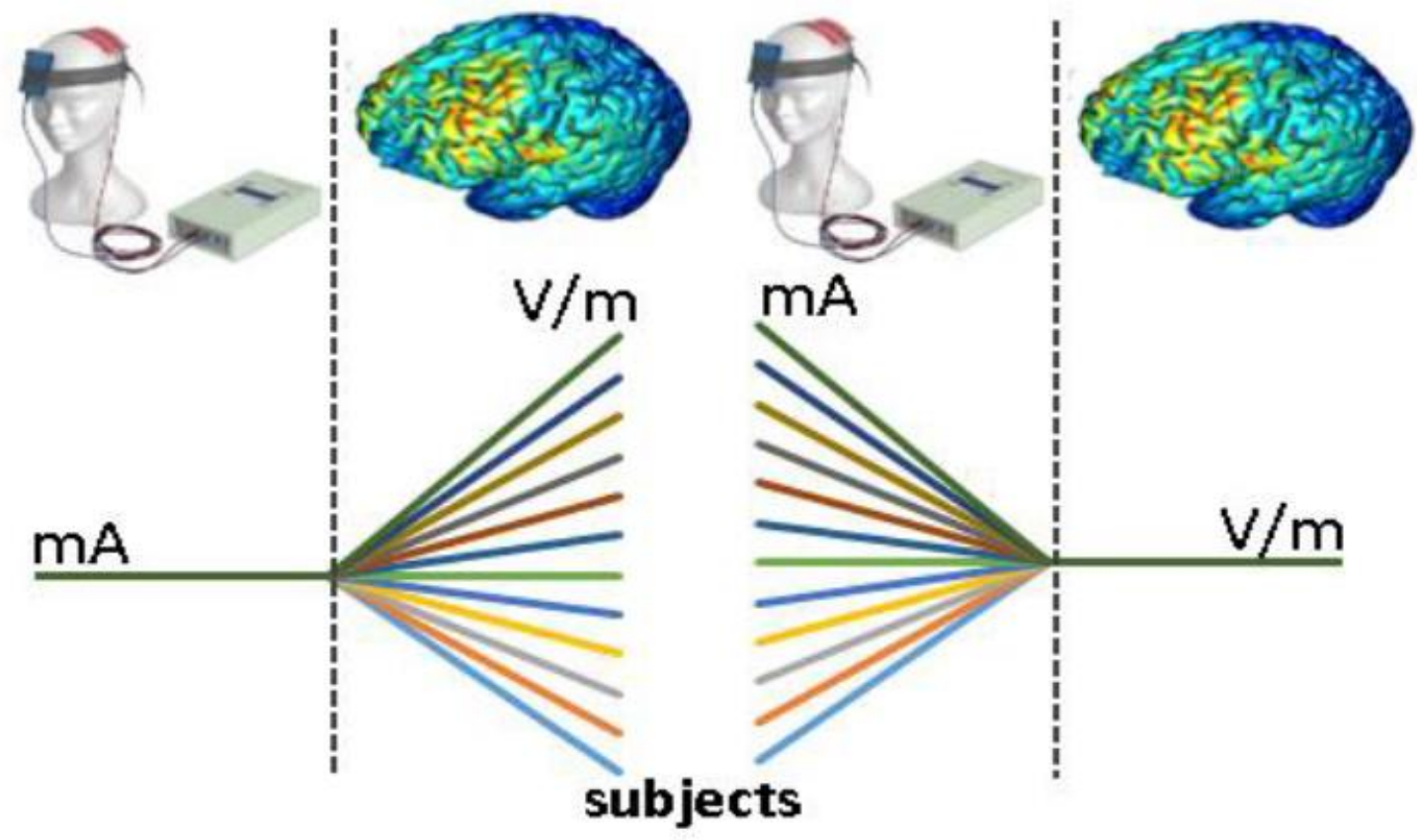

B Fixed electrical field inside the brain

Figure 1: A. A fixed stimulator output will result in highly variable current delivery inside the brain across individuals. $B$. By contrast, current delivery in a target regions is comparable across individuals when adjusting stimulator output individually, based on current flow model estimates from individual MRI scans.

Two recent studies $[6,8]$ now provide important assurance that current flow models indeed do a good job in estimating how much and where to tES delivers current in the brain of an individual. Opitz and colleagues measured the spatial and temporal distribution of the electrical fields generated by 
alternating transcranial current stimulation (tACS), using implanted electrodes in cebus monkeys and patients with epilepsy undergoing intracranial recordings prior to neurosurgical treatment[8]. The current strengths measured in the brain exhibited substantial inter-individual differences, but these matched well with estimates from previous modelling studies. A more direct comparison of estimated and actual current was conducted by Huang and colleagues[6]. These authors directly measured the magnitude and spatial distribution of electrical fields in human epilepsy patients with implanted electrodes, and compared these with the estimates from current flow models obtained from structural MRIs of the same patients. Encouragingly, the predicted electrical fields across the brain correlated highly with measured electrical fields. In both studies, the measured electrical fields in the brain were in the order of $0.2-0.5 \mathrm{~V} / \mathrm{m}$ for stimulator outputs of $1-2 \mathrm{~mA}$, thus being at the lower bound of animal studies. Both studies also highlight that currents of this magnitude do not just occur under the stimulation electrodes, but are widely distributed and can include deep brain structures and periventricular white matter.

These results provide overdue first validations of current flow models, and their ability to control and individualize the dose of current actually delivered to the brain. It is easy to imagine that the inevitable variability in current delivered to the brain across individuals has so far been a key determinant for the variable effects of tES. What is puzzling, however, is that in most discussions about the variable effects of tES, this key point is absent $[4,5,10]$. As long as we do not know how much current is effectively delivered in an individual, the debate about the sources of variable responses to tES (including, for example, cortical state dependencies, individual traits, or genetic variables) is like posting a letter without a stamp nor address, and hoping it will arrive at its destination. With the recent validation of current flow models[6, 8], the field is now in a position to better control variance arising from dose differences across individuals, and thereby identify the true biological causes of variable tES effects. 
But such efforts would rest on another and yet untested question: would individualizing dose based on these models actually reduce physiological and behavioural inter-subject variability to tES? Or do these models merely provide expensive and complex MRI colouring-in software, which predicts current flow in the brain accurately, but make no difference whatsoever to the variable effects tES might have on physiology and behaviour? It is perplexing that the development of current flow models over the past decade, including recommendations of their use in patients groups with brain lesions, has vastly outpaced attempts to answer these questions, some notable exceptions aside[2].

Answering this may not be easy. Controlling current strength alone may not be sufficient to improve the efficacy of tES, because the direction of current flow with respect to the cortical surface determines the physiological response to stimulation. It is worth recalling that in vitro and in vivo studies in animals neatly control the direction of current flow and intensity, and record neural activity from small circumscribed cortical patches without the complexity of cortical folding. It remains to be seen whether these results map directly onto human tES applications. The field must therefore now turn to identifying the optimization criteria for the controlled delivery of tES, and whether dose control based on these indeed improves the efficacy of tES.

In providing validation that current flow models are doing a good job in predicting where current flows in the brain, and how much of it gets there, the aforementioned studies $[6,8]$ provide the foundation for individualizing dose delivery of tES. With this, the field can now (re)start addressing what may truly cause variable effects of tES, and whether the controversy about its overall utility in basic and translational research is justified or not. With good models to predict current flow in the brain, efforts must now turn to showing that they are actually useful in improving the efficacy of tES.

References 
1. Datta A, Truong D, Minhas P, Parra LC, Bikson M (2012) Inter-Individual Variation during Transcranial Direct Current Stimulation and Normalization of Dose Using MRI-Derived Computational Models. Front Psychiatry 3:91

2. Edwards D, Cortes M, Datta A, Minhas P, Wassermann EM, Bikson M (2013) Physiological and modeling evidence for focal transcranial electrical brain stimulation in humans: a basis for highdefinition tDCS. Neuroimage 74:266-75

3. Elsner B, Kugler J, Pohl M, Mehrholz J (2016) Transcranial direct current stimulation (tDCS) for improving activities of daily living, and physical and cognitive functioning, in people after stroke. Cochrane Database Syst Rev 3:CD009645

4. Hill AT, Fitzgerald PB, Hoy KE (2016) Effects of Anodal Transcranial Direct Current Stimulation on Working Memory: A Systematic Review and Meta-Analysis of Findings From Healthy and Neuropsychiatric Populations. Brain Stimul 9:197-208

5. Horvath JC, Vogrin SJ, Carter O, Cook MJ, Forte JD (2016) Effects of a common transcranial direct current stimulation (tDCS) protocol on motor evoked potentials found to be highly variable within individuals over 9 testing sessions. Exp Brain Res 234:2629-42

6. Huang Y, Liu AA, Lafon B, Friedman D, Dayan M, Wang X, Bikson M, Doyle WK, Devinsky O, Parra LC (2017) Measurements and models of electric fields in the in vivo human brain during transcranial electric stimulation. Elife 6:e18834

7. Laakso I, Tanaka S, Koyama S, De S, V, Hirata A (2015) Inter-subject Variability in Electric Fields of Motor Cortical tDCS. Brain Stimul 8:906-13

8. Opitz A, Falchier A, Yan CG, Yeagle EM, Linn GS, Megevand P, Thielscher A, Deborah AR, Milham MP, Mehta AD, Schroeder CE (2016) Spatiotemporal structure of intracranial electric fields induced by transcranial electric stimulation in humans and nonhuman primates. Sci Rep 6:31236

9. Rush S, Driscoll DA (1968) Current distribution in the brain from surface electrodes. Anesth Analg 47:717-23

10. Tremblay S, Larochelle-Brunet F, Lafleur LP, El MS, Lepage JF, Theoret H (2016) Systematic assessment of duration and intensity of anodal transcranial direct current stimulation on primary motor cortex excitability. Eur J Neurosci 44:2184-90 
